\title{
BMJ Open Efficacy of two injection-site localisation techniques for botulinum toxin injections: a single-blind, crossover, randomised trial protocol among adults with hemiplegia due to stroke
}

\author{
Claire Morel, ${ }^{1,2}$ Isabelle Hauret, ${ }^{1,3}$ Nicolas Andant, ${ }^{4}$ Armand Bonnin, ${ }^{1,2}$ \\ Bruno Pereira, ${ }^{4}$ Emmanuel Coudeyre ${ }^{1,2,5}$
}

To cite: Morel C, Hauret I, Andant N, et al. Efficacy of two injection-site localisation techniques for botulinum toxin injections: a singleblind, crossover, randomised trial protocol among adults with hemiplegia due to stroke. BMJ Open 2016;6: e011751. doi:10.1136/ bmjopen-2016-011751

- Prepublication history and additional material is available. To view please visit the journal (http://dx.doi.org/ 10.1136/bmjopen-2016011751)

Received 2 March 2016 Revised 2 September 2016 Accepted 8 September 2016

CrossMark

For numbered affiliations see end of article.

\section{Correspondence to}

Emmanuel Coudeyre;

ecoudeyre@chu-

clermontferrand.fr

\section{ABSTRACT}

Introduction: Botulinum toxin injections are an effective treatment for limb spasticity following stroke. Different tracking techniques are used for this purpose: palpation, electrostimulation, electromyography and ultrasound. Yet very few studies have compared these different techniques, and none has successfully proved the superior efficacy of ultrasound-guided injections compared to another tracking method. The primary objective of our study was therefore to compare the efficacy of botulinum toxin injections depending on the tracking technique used: ultrasound versus electrostimulation.

Methods and analysis: This is a clinical, singlecentre, prospective, interventional, single-blind, crossover, randomised trial. In total, 30 patients aged between 18 and 80 years presenting with triceps surae spasticity (evaluated $>1$ on the modified Ashworth scale) associated with hemiplegia sequelae due to stroke will be included. The patients will be selected among those who attend for consultation the Physical Medicine and Rehabilitation Department of the Clermont-Ferrand University Hospital. One group will receive the abobotulinumtoxinA (BoNT-A) injection guided by electrostimulation then ultrasound, and the second group's botulinum toxin injections will be guided by ultrasound then electrostimulation. For each patient, the duration of study participation is 5 months. The primary end point is variation in passive ankle dorsiflexion range of motion at slow and high speeds (Tardieu scale) with the knee straight.

Ethics and dissemination: This study received ethics approval form the CPP of Rhônes-Alpes region. Results will be published in a peer-reviewed journal.

Trial registration number: NCT01935544; pre-results.

\section{INTRODUCTION}

The management of muscle spasticity is a major challenge in hemiplegia following a
Strengths and limitations of this study

- The management of muscle spasticity proves to be a major challenge in hemiplegia following a stroke, with botulinum toxin injections constituting the first-line treatment for local or loco-regional spasticity.

- Concerning the limitations, it is a prospective study with inherent risks due to this type of studies such as lost to follow-up bias.

- No study has as definitely yet successfully proved the benefits of ultrasound-guided botulinum toxin injections in terms of efficacy and patient comfort compared to other guiding techniques.

stroke, with botulinum toxin injections constituting the first-line treatment for local or loco-regional spasticity. ${ }^{1}$

Yet there is a range of techniques involving different methods for injection and tracking. The most commonly used tracking techniques are anatomy palpation, electrostimulation, electromyography and, more recently, ultrasound. Palpating children to guide injection is not reliable, particularly when the deeper muscles are concerned (eg, only $12 \%$ successfully positioned injections in the tibialis posterior and flexor carpi ulnaris), but also for more superficial muscles $(22 \%$ failure rate for the gastrocnemius).$^{2}$ For children, injection guided via electromyogram (EMG) is not always appropriate, when there is difficulty obtaining active or passive muscle activation, to differentiate muscular activation of a specific muscle from surrounding muscles. ${ }^{3}$ In addition, with this technique, there is no correlation between the extent of spasticity and muscular activity. ${ }^{3}$ One article ${ }^{4}$ shows that neither manual needle placement 
nor electrical stimulation is wholly accurate to inject gastrocnemius muscle of adults with spasticity.

Tracking via ultrasound is widely used in other indications, such as infiltrations in the locomotor system (particularly the tendons and joints) ${ }^{5}$ or anaesthetic nerve blocks. ${ }^{6}$ The primary advantages of ultrasound-guided botulinum toxin injection are that tracking is painless, ${ }^{3}$ fast, ${ }^{7}$ more precise $^{3}$ and thus safer, avoiding complications associated with subcutaneous, intravascular or too-deep injections. ${ }^{8}$

A comparative study has assessed the efficacy of ultrasound-based tracking with an electrostimulation-based technique. ${ }^{9}$ The authors evaluated 32 children presenting with cerebral palsy sequelae, who were divided into two groups. All received botulinum toxin injection into the gastrocnemius, which was guided by either ultrasound or electrostimulation depending on the group. The techniques were evaluated based on three different scales: the Ashworth (see online supplementary appendix 1), Tardieu (see online supplementary appendix 2 ) ${ }^{10}$ selective motor control (SMC) ${ }^{9}$ and Physician Rating scales. The authors observed a non-significant improvement in spasticity, assessed by the Ashworth and Tardieu scales, at 3 months postinjection, in the group treated with ultrasound-guided injections. In contrast, the electrostimulation-guided group showed non-significant improvement in motor control of the antagonistic muscles. The only significant differences revealed were improvements in walking pattern and foot-to-ground contact in the ultrasound-guided group. Nonetheless, the numerous controversial methodological choices made by the authors limited the relevance of these results.

There have also been two comparative studies evaluating the efficacy of ultrasound-guiding with that of techniques using electrostimulation or anatomy palpation. ${ }^{11} 12$

The first study, ${ }^{11}$ conducted in 2012, compared these three injection-guiding techniques in the lower limbs. The trial involved 49 patients presenting with lower-limb spasticity following stroke, who were randomised into three groups, the first group receiving injections guided by anatomy palpation, the second group by electrostimulation and the last group by ultrasound. All received a botulinum toxin injection into the gastrocnemius, administered by the same physician. The investigator, who was blinded to the injection type, evaluated each patient on inclusion and at 1 month. The patients were forbidden from undergoing any form of physical therapy within the 3 months preceding the study and during its entirety. Results of the Ashworth and Tardieu scales were assessed for all, along with passive dorsiflexion of the foot. The authors reported significantly improved passive dorsiflexion of the foot in the ultrasound-guided injection group compared to the electrostimulation-guided group. Moreover, results of the Ashworth scale were significantly improved 1 month following botulinum toxin injection in the ultrasound-guided group compared to the group where anatomy palpation was used.

The second study ${ }^{12}$ was conducted in 2013 and assessed upper-limb spasticity in 60 patients who had suffered from strokes. As in the above-described study, these patients were randomised into three groups of 20 each in order to compare the three injection-guiding techniques: ultrasound, electrostimulation, and anatomy palpation. Two injections were administered in at least two of the following muscles: flexor carpi ulnaris, flexor carpi radialis, flexor digitorum superficialis and flexor digitorum profundus. The same physician, experienced with using botulinum toxin under ultrasound-guiding, administered all the injections. An investigator who was blinded to the injection type assessed each patient at the beginning and 4 weeks into the study. The patients were forbidden from undergoing any type of physical therapy in the 3 months preceding the study and during its entirety. Results of the Ashworth and Tardieu scales were assessed, along with passive dorsiflexion of the wrist and fingers. One month following injection, scores of the modified Ashworth scale significantly improved in the group having undergone ultrasound-guided injection compared to the group tracked using anatomy palpation, as did scores of the Tardieu scale and passive mobilisations. In contrast, the authors found no significant differences between ultrasound- and electrostimulationguiding for the different evaluations.

In both of these studies, the authors described limitations consisting of the absence of functional evaluation of the upper or lower limbs, owing to the short follow-up rendering this assessment difficult to implement, as well as of the injections being administered by only one physician experienced with ultrasound-guided injection. The authors also indicated that body mass index was not taken into account in their studies, despite obesity potentially constituting a limitation to the accurate assessment of anatomical landmarks.

In a literature review, ${ }^{13}$ all four guiding techniques (anatomy palpation, electromyography (EMG), electrostimulation and ultrasound) were compared, with advantages and disadvantages outlined for each. The authors retrieved and analysed 15 articles, concluding that injection guided by anatomy palpation required no equipment and only a small-sized needle. Yet deep or slighter muscles were more difficult to access. In addition, while EMG enabled the toxin to be injected closest to the motor end-plate, this technique could, however, not guarantee that the needle was actually in the target muscle. As for the electrostimulation-guided technique, its primary advantage appeared to be its precise localisation capacity. Despite this, it can take a long time to perform and require more training than the EMG and anatomy palpation techniques. Finally, ultrasound was found to enable the real-time visualisation of the needle's progression while avoiding certain structures like blood vessels or nerves, among other advantages. In addition, the needle used in this technique was finer and thus less painful. On the other hand, this technique was highly dependent on the operator's skill, potentially requiring the presence of an assistant for beginners.

All in all, guiding injections by anatomy palpation thus appears to be the least precise technique. The other 
guiding techniques appear to offer superiority, in terms of precision and thus efficacy, although further studies must be conducted in order to determine which technique achieves the best clinical results.

Another literature review ${ }^{14}$ evaluated the impact of the different injection-guiding techniques on the efficacy of botulinum toxin when treating not only spasticity but also dystonia. This review covered 10 studies, 7 of which were randomised. The authors reported a high level of evidence (Grade A) that instrument-based guiding, that is, ultrasound, electrostimulation or electromyography, was more effective than manual guiding in the treatment of upper-limb spasticity, spastic equinus following stroke in adults and cerebral palsy in children. The review's conclusions were that no instrument-based guiding technique proved superior to another. At the present time, no recommendation can be made in terms of choosing the optimal guiding technique, although ultrasound nevertheless appears to be more effective than electrostimulation in spastic equinus treatment following stroke in adults (passive mobilisation of the ankle). ${ }^{11}$

No study has as definitely yet successfully proved the benefits of ultrasound-guided botulinum toxin injections in terms of efficacy and patient comfort compared to other guiding techniques.

\section{METHODS/DESIGN}

\section{Objective}

Our main objective is to compare the efficacy of botulinum toxin injections in terms of guiding technique: ultrasound versus electrical stimulation in patients with hemiplegia due to stroke.

The secondary objective is to demonstrate that ultrasound guidance is a less painful localisation technique.

\section{Study design}

This prospective, randomised, single-centre, single-blind, crossover study will be conducted in patients with chronic stroke ( $>6$ months) presenting with triceps surae spasticity. Severity of the ambulation deficit was considered by using the Functional Ambulation Classification modified, and stratification was made on ambulation. The patients will receive two injections; each administered using a different guiding technique. Randomisation will determine which technique will be used in the first and second instances. The patients will be selected among those who attend for consultation at the Physical Medicine and Rehabilitation Department of the Clermont-Ferrand University Hospital. The botulinum toxin injections and assessments will take place in the same department. The study will last 5 months for each patient. This study does not present a major risk for the participants. The main potential disadvantages to the treatment are injection pain or side effects from the botulinum toxin (increased motor deficit or dysphagia).

The study design is presented in figure 1.

\section{Randomisation}

The patients will be randomly assigned to one of the above-described groups by means of a Latin square design in order to balance out the group numbers.

\section{Study description}

The patients preselected during consultation at the Physical Medicine and Rehabilitation Department of the Clermont-Ferrand University Hospital will be handed a letter containing information on the study protocol. They will then have 1 month to grant their consent, should they wish and be included at their next consultation.

The following data will then be collected for each patient: age, gender, time since stroke, side affected by the cerebral lesion, current treatments and dosages (for managing spasticity and pain), date of the first botulinum toxin injection and severity of deficit (functional walking scale).

The initial assessment of the patients included in the study will be performed just prior to the first injection. This evaluation will be clinical (assessment of the triceps surae spasticity based on the Tardieu and modified Ashworth scales) and instrument based (walking speed using GAITRite, CIR Systems, Sparta, New Jersey, USA).

The first injection will be administered in the outpatient clinic by a therapist with injection experience of over 3 years, guided using ultrasound or electrostimulation, depending on the group. We use Dantec Clavis for electrostimulation injection and Sonosite Edge with a 6$13 \mathrm{MHz}$ probe for ultrasound injection. The clinical investigator will randomise the patients and then administer the injection according to the guiding method assigned. In total, 500 units of BoNT-A (Dysport) will be injected into four separate areas of the triceps surae to have a good reproducibility. Further injections will be administered into other muscle groups, if necessary. The total dose for this injection will be minus 1500 BoNT-A units. Any pain experienced during the injection will be assessed by means of vertical visual analogue scales, and the time required for tracking and administering the injection will be recorded.

The second injection will be administered 4 months after the first injection, also in the outpatient clinic. The procedure will be identical to the first injection apart from the tracking method used on this occasion which will differ from that used for the first injection.

The two follow-up visits will take place 1 month after each botulinum toxin injection. Each patient will be asked to attend the clinic for consultation so as to allow the efficacy of the injection to be assessed. This assessment will be clinical (assessment of the spasticity of the triceps surae by means of the Tardieu and modified Ashworth scales) and instrument based (walking speed using GAITRite). Each follow-up visit will be performed by an investigator (physiotherapist) blinded to the tracking technique. The patients are also told to hide the kind of injection they received. After each injection, 
Figure 1 Flow diagram showing the different stages of the protocol.

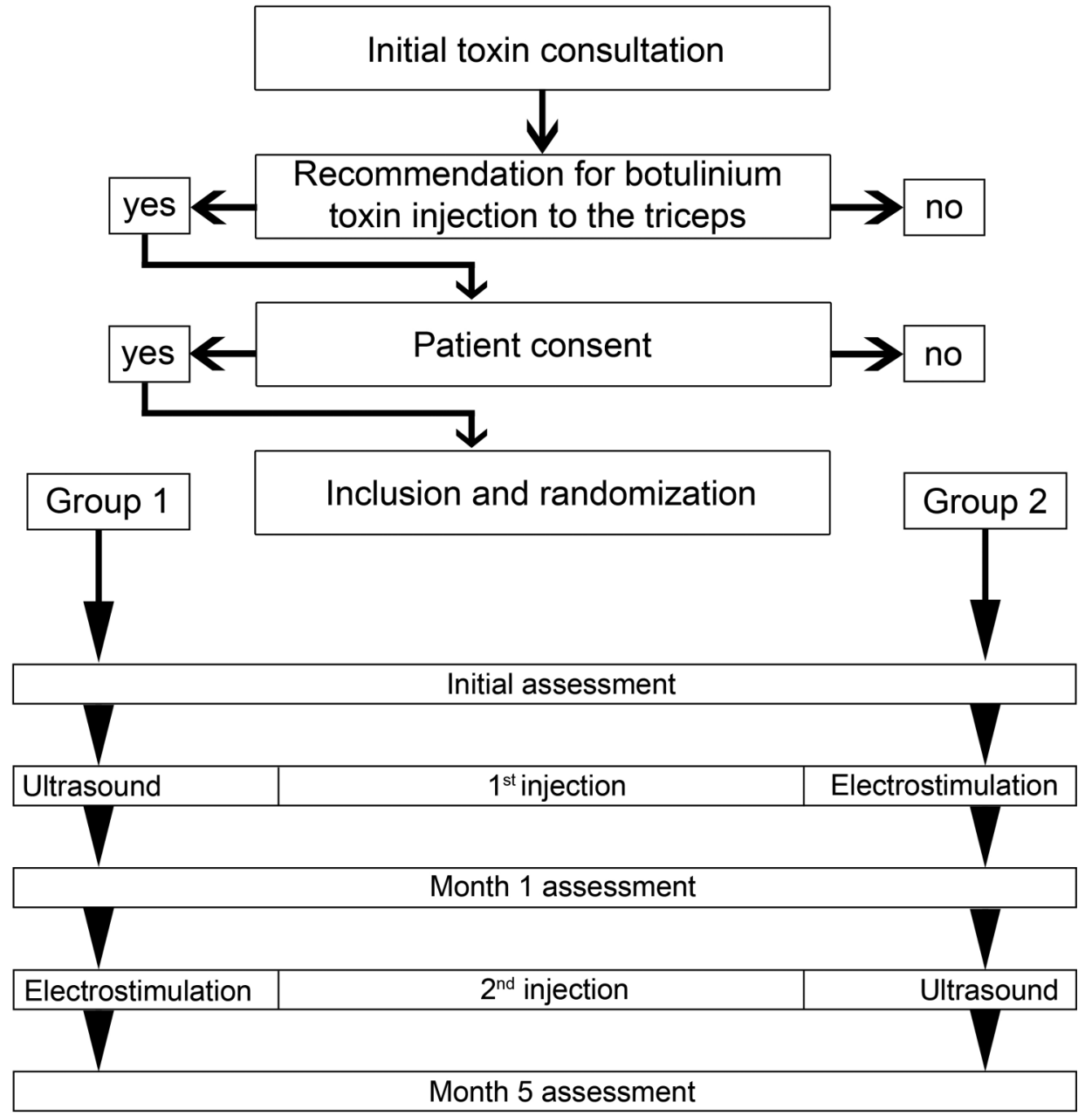

patients were told to continue their regular physiotherapy.

This study is actually ongoing, and the investigators are currently still collecting data. The contents of the manuscript have not been submitted or published elsewhere.

\section{Patients}

The inclusion criteria are as follows: age 18-80 years, hemiplegic sequelae of stroke, triceps surae spasticity evaluated $>1$ on the modified Ashworth scale and ability to provide written consent. The exclusion criteria are as follows: injection of botulinum toxin dating from over 3 months; previous ultrasound-guided injection of botulinum toxin; swallowing impairment; ongoing antivita$\min \mathrm{K} \quad(\mathrm{AVK})$ anticoagulation treatment with international normalised ration (INR) > 3 during 1 week before randomisation; ongoing aminoglycoside treatment; general anaesthesia with planned curare injection during study participation; implant with a pacemaker; history of ankle arthrodesis; other contra-indication for botulinum toxin injection: myasthenia gravis, pregnancy or breast feeding; and patient included in other trials. The indication for botulinum toxin injection to the upper limb will not constitute a non-inclusion criterion for this study.

\section{Evaluation}

The primary end point is variation in passive ankle dorsiflexion range of motion at slow and high speeds (Tardieu scale) while keeping the knee straight.

The procedure consists of assessing the angle at which resistance manifests, as well as the intensity of this resistance to mobilisation at slow and fast speeds. ${ }^{15}$ The ankle dorsal flexion angle will thus be measured by means of a goniometer during passive manipulation of the ankle with the knee being kept straight, before and after treatment. For the Tardieu scale, the minimally clinical important change differs according to studies. ${ }^{16}$ The effect size calculation is based on an improvement of 7 angular degrees which is quite important considering regular ankle range of motion from 0 to $50^{\circ} .^{17}$ Concerning the gait analysis, an improvement of $0.2 \mathrm{~m} /$ $\mathrm{s}$ of the gait speed is considered as a minimally clinical important change. ${ }^{18}$

This straight-knee assessment is relevant for simultaneously obtaining measurement of gastrocnemius muscles, which are bi-articular, and the soleus spasticity. 
The Tardieu scale is more sensitive than the commonly used modified Ashworth scale. The latter only consists of five stages, which does not always allow for treatment efficacy to be evaluated. Furthermore, this scale does not take into account the velocity factor during spasticity. ${ }^{16}$ Nevertheless, validation studies pertaining to the Tardieu scale and involving the adult population are scarce in the scientific literature. Moreover, the Tardieu scale is reliable for assessing spasticity in lower-limb muscles of adults with chronic neurologic injuries. ${ }^{19}$

Assessing the difference in the range of motion between slow and fast speeds is relevant because this takes into account not only the spastic component but also any potential tendon retraction.

The principal evaluation criterion will be measured on the day of injection and at the Month 1 assessment.

The secondary end points are as follows:

- other components of the 'Tardieu scale': quality of muscle reaction $(\mathrm{X})$ at slow and fast speeds, as well as angle of apparition of the muscle reaction $(\mathrm{Y})$ at slow and fast speeds,

- assessment of the triceps surae spasticity on the modified Ashworth scale,

- walking speed,

- extent of pain at the injection site using a visual analogue scale,

- duration of tracking and injection.

\section{Statistical considerations}

To date, only one comparative study focused on the protocol's topic has been published. ${ }^{17}$ Therefore, if scientific literature data provide information on the statistical variability of ankle dorsiflexion range of motion at slow and high speeds obtained using the Tardieu scale for patients having suffered from stroke, ${ }^{17}$ exhibiting an SD around $8.5^{\circ}$, proposing an expected difference between the two treatments (ultrasound vs electrical stimulation) proves challenging. In addition, in order to highlight the efficacy of botulinum toxin injections in terms of guiding technique, namely ultrasound versus electrical stimulation, sample size estimation was based on statistical power simulations in relation to recruitment capacity. To demonstrate a minimum difference of 7.12 between ultrasound and electrical stimulations, with an effect size of 0.8 (so, an expected SD of difference at $8.9^{\circ}$ ), 15 patients per sequence (ultrasound stimulation then electrical vs electrical stimulation then ultrasound) will be needed for a two-sided Type I error at $5 \%$, a statistical power of $80 \%$, an intra-individual correlation coefficient equals 0.5 (owing to the crossover design) and no carry-over effect assumed.

Statistical analysis will be performed on an intention-to-treat basis using the Stata software (V.13, StataCorp, College Station, Texas, USA) for a two-sided Type I error at $\alpha=5 \%$. The patient characteristics will be described by numbers and associated percentages for categorical data. For quantitative parameters, mean (SD) or median (IQR) values will be calculated and presented according to statistical distribution. The assumption of normality will be studied by the Shapiro-Wilk test. The primary end point, namely variation in the ankle dorsiflexion range of motion at slow and high speeds obtained using the Tardieu scale, will be compared between the groups using a repeated analysis of variance (ANOVA) for crossover designs while taking into account the following effects: treatment group (ultrasound vs electrical stimulation), sequence, participant (as random effect) and carry-over. A particular focus will be given to the interaction "sequence $\mathrm{x}$ treatment (ultrasound vs electrical stimulation)". A sensitivity analysis will be proposed to determine the nature of missing data and to apply the more appropriate imputation approach as multiple imputation. If this test proves significant, the statistical analysis will only cover the first period of this crossover study. The normality of residuals will be studied, as described previously. When end points do not assume the normality assumption, a nonparametric paired test like the Wilcoxon will be proposed. Analyses concerning the secondary end points quality of muscle reaction (X) at slow and fast speeds, the angle of the muscle reaction apparition $(\mathrm{Y})$ at slow and fast speeds, assessment of the triceps surae spasticity on the modified Ashworth scale, walking speed and extent of pain at the injection site using a visual analogue scale, along with the duration of tracking and injection will be studied in a similar way as the primary end point. For categorical parameters, the StuartMaxwell test for paired data or generalised linear mixed model taking into account the above-mentioned effects will be applied. Concerning non-crossover comparisons, usual statistical tests will be performed: Student's t-test or the Mann-Whitney test if the conditions of t-test are not met (normality or homoscedasticity verified using the Fisher-Snedecor test) for quantitative parameters and $\chi^{2}$ test or Fisher's exact test for categorical variables, if appropriate. As discussed by Feise,${ }^{20}$ adjustment of Type I error $(\alpha)$ will not be proposed systematically, but on a case-by-case basis in the light of clinical considerations rather than statistical ones only.

\section{DISCUSSION}

The various comparative studies currently available ${ }^{9} 1112$ have demonstrated that instrument-guided procedures, such as electrostimulation and ultrasound, improve the efficacy of botulinum toxin injections compared to that obtained by means of simple anatomy palpation, in line with current recommendations for good clinical practices.

Ultrasound enables us to visualise in real time the needle's progress, resulting in a precise localisation of the target muscle, while avoiding certain structures like blood vessels and nerves. In addition, this technique allows a passive manipulation of the limb part under study in order to distinguish the muscular body of the 
target muscle from that of other adjacent muscular structures. $^{11}$

Ultrasound-guided botulinum toxin injection can be subject to the same limitations inherent to ultrasound itself. The technique is highly dependent on the skills of the operator, who needs to be experienced, thus requiring further investment in terms of training and equipment. Additionally, the structural evolution of spastic muscles, as fatty infiltration and, in particular, fibrous involution, alters the ultrasound features of the muscle, rendering it at times difficult to distinguish from the different adjacent muscles. ${ }^{21} 22$

The substantial cost of ultrasound equipment no longer appears to represent an obstacle to using this guiding technique. It is now possible to directly employ different ultrasound waves with a digital tablet, thus considerably reducing the equipment costs.

With regard to the guiding speed of the different techniques, the literature currently provides contradictory views. The Berweck team ${ }^{7}$ demonstrated that the mean time of muscle localisation and injection was only $5 \mathrm{~s}$ for superficial muscles and $30 \mathrm{~s}$ for deeper ones when using ultrasound. On the other hand, the Henzel study ${ }^{21}$ in 2010 reported an average increase of 5-10 min in procedure time when adding ultrasound-guiding to usual guiding techniques. If ultrasound-guided injection was concretely proved to be faster than other methods, this could represent a particular advantage for children and poorly compliant adults displaying low tolerance for procedures involving prolonged immobilisation. ${ }^{11}$

In this study, we hypothesise that botulinum toxin injections guided by ultrasound are more efficacious than those using electrostimulation, with the triceps surae spasticity as primary evaluation criterion. In addition, we also seek to prove that ultrasound-guided botulinum toxin injections are less painful than those administered using electrostimulation and that the time needed for localising and injecting is shorter for the former.

The expected benefit for the patient is thus a more efficacious injection and consequently reduced spasticity of the triceps surae. The benefits of ultrasound-guided injection compared to that of electrostimulation-guided injection consist of reduced tracking and injection times, in addition to reduced pain on injection.

This study's objective is to improve the techniques pertaining to guiding and injection. When injecting botulinum toxin, it is, in fact, all the more crucial to be as precise as possible in order to ensure the best efficacy in the target muscles while avoiding any unwanted effects that could arise in relation with toxin diffusion or intravascular injection. For this reason, it is highly desirable to use the most reliable guiding method possible. Furthermore, toxin injection can be a painful procedure, particularly for certain patients suffering from hyperaesthesia or cognitive disorders, meaning a guiding technique that enables the highest tolerance is all the more crucial.
Author affiliations

${ }^{1}$ Service de Médecine Physique et de Réadaptation; CHU Clermont-Ferrand, Clermont-Ferrand, France

${ }^{2}$ Université Clermont Auvergne, Clermont-Ferrand, France

${ }^{3}$ Centre médical Etienne Clémentel, Enval, France

${ }^{4}$ Biostatistics Unit, Délégation Recherche Clinique \& Innovation (DRCI), $\mathrm{CHU}$

Clermont-Ferrand, Clermont-Ferrand, France

${ }^{5}$ INRA, Unité de Nutrition Humaine (UNH, UMR 1019), Clermont-Ferrand, France

Contributors BP involved in data analysis, critical revision and final approval of the manuscript. CM involved in manuscript writing, critical revision and final approval of the manuscript. EC involved in conception and design, analysis and interpretation, manuscript writing, critical revision and final approval of the manuscript. $\mathrm{HI}$ involved in conception and design, data collection, analysis and interpretation, manuscript writing, critical revision and final approval of the manuscript. NA involved in study setup, critical revision and approval of the final manuscript. $A B$ involved in English reviewing and critical revision.

Funding This work was supported by CHU Clermont-Ferrand, and IPSEN provided funding for the ultrasound.

Competing interests None declared

Patient consent Obtained.

Ethics approval The study protocol, patient information and patient consent form, along with the case report form, have been submitted to the ethics committee of the Rhône-Alpes region (Comité de Protection des Personnes Rhône-Alpes, CPP Sud-Est I). The CPP's favourable assessment was transmitted to the study sponsor and ANSM (l'Agence nationale de sécurité des medicaments et des produits de santé-the French National Agency of Medicine and Health Products Safety) on 29 November 2012.

Provenance and peer review Not commissioned; externally peer reviewed.

Open Access This is an Open Access article distributed in accordance with the Creative Commons Attribution Non Commercial (CC BY-NC 4.0) license, which permits others to distribute, remix, adapt, build upon this work noncommercially, and license their derivative works on different terms, provided the original work is properly cited and the use is non-commercial. See: http:// creativecommons.org/licenses/by-nc/4.0/

\section{REFERENCES}

1. Yelnik AP, Simon O, Bensmail D, et al. Drug treatments for spasticity. Ann Phys Rehabil Med 2009;52:746-56.

2. Chin TYP, Nattrass GR, Selber P, et al. Accuracy of intramuscular injection of botulinum toxin a in juvenile cerebral palsy: a comparison between manual needle placement and placement guided by electrical stimulation. J Pediatr Orthop 2005;25:286-91.

3. Schroeder AS, Berweck S, Lee SH, et al. Botulinum toxin treatment of children with cerebral palsy-a short review of different injection techniques. Neurotox Res 2006;9:189-96.

4. Picelli $\mathrm{A}$, Bonetti $\mathrm{P}$, Fontana $\mathrm{C}$, et al. Accuracy of botulinum toxin type A injection into the gastrocnemius muscle of adults with spastic equinus: manual needle placement and electrical stimulation guidance compared using ultrasonography. J Rehabil Med 2012;44:450-2.

5. Adler RS, Sofka CM. Percutaneous ultrasound-guided injections in the musculoskeletal system. Ultrasound Q 2003;19:3-12.

6. Walker KJ, McGrattan K, Aas-Eng K, et al. Ultrasound guidance for peripheral nerve blockade. Cochrane Database Syst Rev 2009;(4): CD006459.

7. Berweck S, Schroeder AS, Fietzek UM, et al. Sonography-guided injection of botulinum toxin in children with cerebral palsy. Lancet 2004;363:249-50

8. Berweck S, Heinen F. Use of botulinum toxin in pediatric spasticity (cerebral palsy). Mov Disord Off J Mov Disord Soc 2004;19(Suppl 8) S162-167.

9. Kwon J-Y, Hwang JH, Kim J-S. Botulinum toxin A injection into calf muscles for treatment of spastic equinus in cerebral palsy: a controlled trial comparing sonography and electric stimulation-guided injection techniques: a preliminary report. Am J Phys Med Rehabil Assoc Acad Physiatr 2010;89:279-86. 
10. Gracies J-M, Burke K, Clegg NJ, et al. Reliability of the Tardieu scale for assessing spasticity in children with cerebral palsy. Arch Phys Med Rehabil 2010;91:421-8.

11. Picelli $A$, Tamburin $S$, Bonetti $P$, et al. Botulinum toxin type $A$ injection into the gastrocnemius muscle for spastic equinus in adults with stroke: a randomized controlled trial comparing manual needle placement, electrical stimulation and ultrasonography-guided injection techniques. Am J Phys Med Rehabil Assoc Acad Physiatr 2012;91:957-64.

12. Picelli A, Lobba D, Midiri A, et al. Botulinum toxin injection into the forearm muscles for wrist and fingers spastic overactivity in adults with chronic stroke: a randomized controlled trial comparing three injection techniques. Clin Rehabil 2014;28: 232-42.

13. Walker HW, Lee MY, Bahroo LB, et al. Botulinum toxin injection techniques for the management of adult spasticity. $P M R$ 2015;7:417-27.

14. Grigoriu A-I, Dinomais M, Rémy-Néris O, et al. Impact of injection-guiding techniques on the effectiveness of botulinum toxin for the treatment of focal spasticity and dystonia: a systematic review. Arch Phys Med Rehabil 2015;96:2067-78.

15. Ben Smaïl D, Kiefer C, Bussel B. Clinical evaluation of spasticity. Neurochirurgie 2003:49(2-3 Pt 2):190-8.
16. Haugh AB, Pandyan AD, Johnson GR. A systematic review of the Tardieu scale for the measurement of spasticity. Disabil Rehabil 2006;28:899-907.

17. Singh $\mathrm{P}$, Joshua $\mathrm{AM}$, Ganeshan $\mathrm{S}$, et al. Intra-rater reliability of the modified Tardieu scale to quantify spasticity in elbow flexors and ankle plantar flexors in adult stroke subjects. Ann Indian Acad Neurol 2011;14:23-6.

18. Lewek MD, Randall EP. Reliability of spatiotemporal asymmetry during overground walking for individuals following chronic stroke. $J$ Neurol Phys Ther 2011;35:116-21.

19. Ben-Shabat E, Palit M, Fini NA, et al. Intra- and interrater reliability of the modified Tardieu scale for the assessment of lower limb spasticity in adults with neurologic injuries. Arch Phys Med Rehabil 2013;94:2494-501.

20. Feise RJ. Do multiple outcome measures require $p$-value adjustment? BMC Med Res Methodol 2002;2:8.

21. Henzel MK, Munin MC, Niyonkuru C, et al. Comparison of surface and ultrasound localization to identify forearm flexor muscles for botulinum toxin injections. PM R 2010;2:642-6.

22. Picelli $A$, Bonetti $P$, Fontana $C$, et al. Is spastic muscle echo intensity related to the response to botulinum toxin type $A$ in patients with stroke? A cohort study. Arch Phys Med Rehabil 2012;93:1253-8. 